\title{
Secernin-1 is a novel phosphorylated tau binding protein that accumulates in Alzheimer's disease and not in other tauopathies
}

Geoffrey Pires', Sacha McElligott', Shiron Drusinsky', Glenda Halliday ${ }^{3}$, Marie-Claude Potier ${ }^{4}$, Thomas Wisniewski ${ }^{1,2^{*}}$ and Eleanor Drummond ${ }^{1,3^{*}}$

\begin{abstract}
We recently identified Secernin-1 (SCRN1) as a novel amyloid plaque associated protein using localized proteomics. Immunohistochemistry studies confirmed that SCRN1 was present in plaque-associated dystrophic neurites and also revealed distinct and abundant co-localization with neurofibrillary tangles (NFTs). Little is known about the physiological function of SCRN1 and its role in Alzheimer's disease (AD) and other neurodegenerative diseases has not been studied. Therefore, we performed a comprehensive study of SCRN1 distribution in neurodegenerative diseases. Immunohistochemistry was used to map SCRN1 accumulation throughout the progression of AD in a cohort of 58 patients with a range of NFT pathology (Abundant NFT, $n=21$; Moderate NFT, $n=22$; Low/No NFT, $n=15$ ), who were clinically diagnosed as having $A D$, mild cognitive impairment or normal cognition. SCRN1 accumulation was also examined in two cases with both Frontotemporal Lobar Degeneration (FTLD)-Tau and ADrelated neuropathology, cases of Down Syndrome (DS) with AD $(n=5)$, one case of hereditary cerebral hemorrhage with amyloidosis - Dutch type (HCHWA-D) and other non-AD tauopathies including: primary age-related tauopathy (PART, $[n=5])$, Corticobasal Degeneration (CBD, $[n=5])$, Progressive Supranuclear Palsy (PSP, $[n=5])$ and Pick's disease (PiD, $[n=4])$. Immunohistochemistry showed that SCRN1 was a neuronal protein that abundantly accumulated in NFTs and plaque-associated dystrophic neurites throughout the progression of AD. Quantification of SCRN1 immunohistochemistry confirmed that SCRN1 preferentially accumulated in NFTs in comparison to surrounding non-tangle containing neurons at both early and late stages of AD. Similar results were observed in DS with AD and PART. However, SCRN1 did not co-localize with phosphorylated tau inclusions in CBD, PSP or PiD. Coimmunoprecipitation revealed that SCRN1 interacted with phosphorylated tau in human AD brain tissue. Together, these results suggest that SCRN1 is uniquely associated with tau pathology in AD, DS and PART. As such, SCRN1 has potential as a novel therapeutic target and could serve as a useful biomarker to distinguish AD from other tauopathies.
\end{abstract}

Keywords: Alzheimer's disease, Tauopathies, Phosphorylated tau, Neurofibrillary tangles, Secernin-1, Protein-protein interaction

\footnotetext{
* Correspondence: Thomas.wisniewski@nyulangone.org;

eleanor.drummond@sydney.edu.au

${ }^{1}$ Center for Cognitive Neurology and Department of Neurology, New York

University School of Medicine, 435 East 30th Street, Rm 1017, New York, NY

10016, USA

Full list of author information is available at the end of the article
}

(c) The Author(s). 2019 Open Access This article is distributed under the terms of the Creative Commons Attribution 4.0 International License (http://creativecommons.org/licenses/by/4.0/), which permits unrestricted use, distribution, and

reproduction in any medium, provided you give appropriate credit to the original author(s) and the source, provide a link to the Creative Commons license, and indicate if changes were made. The Creative Commons Public Domain Dedication waiver (http://creativecommons.org/publicdomain/zero/1.0/) applies to the data made available in this article, unless otherwise stated. 


\section{Introduction}

Alzheimer's disease (AD) is the most common form of dementia. It is characterized by extracellular aggregation of the amyloid- $\beta$ (A $\beta)$ peptide into plaques and intraneuronal accumulation of aggregated and hyperphosphorylated tau (pTau) into neurofibrillary tangles (NFTs) [17, 49]. In physiological conditions, tau is a microtubule-associated protein that promotes microtubule stabilization and axonal transport in the brain [6, $74]$. In the adult brain, there are 6 tau isoforms derived from alternative splicing of exon 2,3 and 10 of the MAPT gene $[6,30]$. These 6 isoforms differ from one another by the absence or presence of two inserts in the $\mathrm{N}$-terminus and by the presence of either three (3R-tau isoforms) or four (4R-tau isoforms) repeats in the microtubule-binding domain $[6,30,35]$. In neurodegenerative diseases, tau is hyper-phosphorylated and undergoes important conformational changes, causing it to aggregate and form lesions in the brain [3, 31, 32, 35]. In $\mathrm{AD}$, both $\mathrm{A} \beta$ and pTau contribute to the development of disease. However, in other neuropathological conditions, dysfunction of tau alone is sufficient to cause dementia [29, 43]. Together, these neurodegenerative diseases are referred to as tauopathies, a group of diseases that includes (but is not limited to) AD, Down Syndrome (DS), Pick's Disease (PiD), Corticobasal Degeneration (CBD), Progressive Supranuclear Palsy (PSP) and Primary age-related tauopathy (PART) [10, 18, 23]. Each type of tauopathy has a distinct clinical phenotype and distinct neuropathology of pTau aggregates that contain different ratios of $3 \mathrm{R}$ and $4 \mathrm{R}$ tau isoforms. pTau aggregates in $\mathrm{AD}, \mathrm{DS}$ and PART contain a mixture of $3 R$ and $4 R$ tau [42], pTau aggregates in CBD and PSP contain only $4 \mathrm{R}$ tau [62] and pTau aggregates in PiD contain only $3 \mathrm{R}$ tau [43]. It has been proposed that the ratio of $3 R: 4 R$ tau is of particular pathological importance as this ratio determines the conformation of pTau aggregates and the associated cofactors, which in turn determines mechanism of disease $[5,16,66]$.

Protein-protein interactions between pTau and surrounding proteins likely mediates the toxic effects of pTau and influences the development of pTau aggregates in tauopathies. Here, we show that Secernin-1 (SCRN1) is a new protein that interacts with pTau in select tauopathies. We first identified SCRN1 as a new protein associated with $\mathrm{AD}$ in our study of the amyloid plaque proteome $[11,12]$. In this study, SCRN1 was selected for validation because it was one of the most abundant novel proteins present in amyloid plaques. Our preliminary IHC studies confirmed that SCRN1 was present in amyloid plaques, but unexpectedly, it was present in plaques with a distribution consistent with dystrophic neurites rather than diffuse distribution throughout amyloid plaques. In these preliminary studies we also observed abundant colocalization of SCRN1 in neurofibrillary tangles, suggesting that it may have an important interaction with pTau in AD. Very little is known about SCRN1 and its function is poorly characterized. SCRN1 is a cytosolic $50-\mathrm{kDa}$ protein that was initially identified as a regulator of exocytosis in mast cells [73]. The majority of previous research studying SCRN1 has examined its role in cancer, where it was found to be overexpressed in a variety of cancers and determined to be a tumor-associated protein that correlated with tumor development and poor prognosis [46, $50,55,67]$. Data mining of transcriptomic and proteomic datasets revealed that SCRN1 is highly expressed in the brain in comparison to other tissue types, both at the mRNA and protein levels [20, 33, 70]. Despite this, we currently have a poor understanding of SCRN1 localization and function in the brain, both in physiological and pathological conditions. SCRN1 overexpression has been observed in the cortex of people with bipolar disorder [59] and in a chick model of retinitis pigmentosa [26]. Recent evidence suggests that SCRN1 may have an important role in regulating endoplasmic reticulum signaling and synaptic vesicle cycling in presynaptic terminals [51], therefore providing new insight into the physiological role of SCRN1 in the brain. Only a few previous studies have described an association between SCRN1 and AD. One study identified SCRN1 as an early marker of neurodegeneration in a transgenic mouse overexpressing human tau [9]. A second study detected SCRN1 in aggregates purified from human AD brain tissue [2]. Recently, SCRN1 was identified as a highly significant CSF biomarker for AD [39]. Together, these three studies strongly hint that SCRN1 may potentially have an important role in AD pathogenesis, perhaps related to synaptic dysfunction, however no studies to date have examined SCRN1 distribution in the human AD brain.

Here, we have performed a comprehensive immunohistochemistry study to characterize SCRN1 throughout the progression of $\mathrm{AD}$ and in a range of other neurodegenerative diseases. We show that SCRN1 is a neuronal protein that abundantly accumulates in NFTs and plaque associated dystrophic neurites throughout the progression of $\mathrm{AD}$ and in $\mathrm{DS}$ and PART. Surprisingly, SCRN1 did not co-localize with pTau-positive glial or neuronal inclusions in CBD, PSP or PiD. Analysis of the interaction of SCRN1 with pTau in AD human brain tissue revealed a significant interaction between SCRN1 and pTau. Together, these results suggest that SCRN1 is uniquely associated with tau pathology in AD, DS and PART. Given its specificity to AD pathology, SCRN1 could serve as a useful AD biomarker and has potential as a novel therapeutic target. 


\section{Methods}

\section{Ethics statement}

All cases used in this study were from ethicallyapproved longitudinally-assessed regional brain donor programs on neurodegenerative diseases. All procedures were performed under protocols approved by the New York University Alzheimer Disease Center, NY, the South Eastern Sydney and Illawarra Local Health District and the Universities of New South Wales and Sydney, Australia. In all cases, written informed consent for research was obtained from the patient or legal guardian, and the material used had appropriate ethical approval for use in this project. All patients' data and samples were coded and handled according to NIH and NHMRC guidelines to protect patients' identities.

\section{Human brain tissue}

All human brain samples with a clinical diagnosis of AD, mild cognitive impairment (MCI) or normal aged controls were obtained from the New York University Alzheimer's Disease Clinical Center (NYU ADC, New York, $\mathrm{NY}$ ) and were classified neuropathologically using an "ABC" score [56]. This cohort of cases was separated into three groups based on NFT load in the hippocampus and neighboring cortex ("High NFT $(++)$ " > 1\% NFT load; $0.01 \%<$ "Moderate NFT (+)" $\leq 1 \%$ NFT load; "Low/ No NFTs $(-)$ " $\leq 0.01 \%$ NFT load). The "High NFT" group $(n=21)$ had a clinical diagnosis of AD. The majority of cases included in the "Moderate NFT" group $(n=22)$ had a clinical diagnosis of MCI. All control cases with no cognitive impairment were in the "Low/No NFT" group $(n=15)$. The DS cohort was selected from donated brain tissue collected at the Institute for Basic Research in Developmental Disabilities (IBR, Staten Island, NY) and all had extensive AD neuropathology. The CBD, PSP, PiD and PART cohorts were selected from donated brain tissue held by the University of Sydney. Individual patient information is included below in Table 1.

\section{Immunohistochemistry}

Fluorescent immunohistochemistry was performed on formalin-fixed paraffin-embedded tissue sections as described previously [12]. Briefly, $8 \mu \mathrm{m}$ thick sections were deparaffinized and rehydrated through a series of xylene and ethanol washes. Antigen retrieval was performed by treatment with $88 \%$ formic acid for $7 \mathrm{~min}$, followed by boiling in citrate buffer $(10 \mathrm{mM}$ sodium citrate, $0.05 \%$ Tween-20; pH 6). Sections were blocked with $10 \%$ normal goat serum, and incubated overnight at $4{ }^{\circ} \mathrm{C}$ with $\alpha$ SCRN1 primary antibody (1:100; LSBio, catalog \#LSC162903) in combination with the anti-pTau antibodies PHF1 (1:200; generously provided by Dr. Peter Davies, Albert Einstein University, NY, NY), MC1 (1:200; provided by Dr. Peter Davies) or AT8 (1:500; Thermo Fisher Scientific; catalog \#MN1020) to label neurofibrillary tangles and dystrophic neurites, RD3 or RD4 antibodies (1:1000; Millipore, catalog \#05-803 and \#05-804) to label specific $3 \mathrm{R}$ or $4 \mathrm{R}$ Tau isoforms, or a combination of anti-A $\beta$ antibodies 4G8 (1:4000; BioLegend; catalog \#800702) and 6E10 (1:4000; BioLegend; catalog \#803001) to label amyloid plaques. Sections were then incubated for $2 \mathrm{~h}$ at room temperature with appropriate fluorescent secondary antibodies (all diluted 1:500, from Jackson ImmunoResearch) and coverslipped (ProLong ${ }^{\mathrm{Tm}}$ Diamond Antifade Mountant, Invitrogen).

\section{Confirmation of antibody specificity}

$\alpha$-SCRN1 antibody specificity was confirmed by antibody pre-absorption. Two micrograms $\alpha$-SCRN1 antibody was pre-absorbed by incubation with recombinant human SCRN1 protein (Novus Biologicals, catalog \#NBP252099 ) at a ratio of $10: 1$ for $1 \mathrm{~h}$ at RT with over-end rotation. Staining intensity of pre-absorbed antibody was compared to non-preabsorbed $\alpha$-SCRN1 antibody that was treated in the same way as the preabsorbed antibody and negative control staining that was not treated with primary antibody. Pre-absorption with SCRN1 protein significantly reduced immunostaining, therefore showing evidence of antibody specificity (Additional file 1: Figure S1).

\section{Quantification of Secernin-1 inside and outside neurofibrillary tangles}

Quantification was performed on two separate cohorts. The first cohort included $n=58$ cases with ADassociated pathology and $n=5$ DS cases. Here, fluorescent imaging of the whole brain section was performed at 20x magnification using a NanoZoomer HT2 (Hamamatsu) whole slide scanner using the same imaging settings for all slides. Four pictures containing the cortex and three pictures containing the hippocampus (capturing CA1, CA2, and CA3) were collected at 4x magnification per case for quantification. The second cohort included $n=5$ cases of CBD, $\mathrm{n}=5$ cases of PSP, $\mathrm{n}=4$ cases of PiD, $n=6$ cases of PART and $n=6$ cases of AD selected from the first cohort for comparison. All pictures were collected at 20X magnification per case using a Zeiss LSM700 Confocal microscope. For hippocampal sections (PART, PiD and AD), four pictures containing the hippocampus (capturing dentate gyrus, CA1, CA2 and CA3) and six pictures containing the adjacent entorhinal and temporal cortex were taken for quantification. For basal ganglia sections (PSP, CBD and AD), four pictures of caudate, four pictures of putamen and two pictures of globus pallidus were collected. Quantification was performed using ImageJ. In each cohort, SCRN1 positive staining was identified as all pixels above a 
Table 1 Individual case information. Cases were recoded according to their PHF1\% load in hippocampus into their respective NFT score (" $-\leq 0.01 \% ; 0.01 \%<"+$ " $\leq 1 \% ; "++">1 \%)$. Hipp = Hippocampus. BG = Basal Ganglia. TCx=Temporal Cortex. ADRC = Alzheimer's disease related changes

\begin{tabular}{|c|c|c|c|c|c|c|}
\hline ID & Diagnosis & $A B C$ score & Sex & Age & NFT score & Region \\
\hline High NFT 1 & $A D$ & $A 3, B 3, C 3$ & $M$ & 89 & ++ & Hipp \\
\hline High NFT 2 & $A D$ & $\mathrm{~A} 3, \mathrm{~B} 3, \mathrm{C} 3$ & $\mathrm{~F}$ & 80 & ++ & Hipp + BG \\
\hline High NFT 3 & $A D$ & $\mathrm{~A} 3, \mathrm{~B} 3, \mathrm{C} 3$ & $\mathrm{~F}$ & 86 & ++ & Hipp \\
\hline High NFT 4 & $A D$ & $\mathrm{~A} 3, \mathrm{~B} 3, \mathrm{C} 3$ & $\mathrm{~F}$ & 98 & ++ & Hipp \\
\hline High NFT 5 & $A D$ & $\mathrm{~A} 3, \mathrm{~B} 3, \mathrm{C} 3$ & $\mathrm{~F}$ & 98 & ++ & Hipp \\
\hline High NFT 6 & $A D$ & $\mathrm{~A} 3, \mathrm{~B} 3, \mathrm{C} 3$ & $\mathrm{~F}$ & 85 & ++ & Hipp \\
\hline High NFT 7 & $A D$ & $\mathrm{~A} 3, \mathrm{~B} 3, \mathrm{C} 3$ & $\mathrm{~F}$ & 81 & ++ & Hipp \\
\hline High NFT 8 & $A D$ & $\mathrm{~A} 3, \mathrm{~B} 3, \mathrm{C} 3$ & $\mathrm{~F}$ & 92 & ++ & Hipp \\
\hline High NFT 9 & $A D$ & $A 3, B 3, C 3$ & $\mathrm{~F}$ & 90 & ++ & Hipp \\
\hline High NFT 10 & $A D$ & $A 3, B 3, C 3$ & $\mathrm{~F}$ & 71 & ++ & Hipp \\
\hline High NFT 11 & $A D$ & $\mathrm{~A} 3, \mathrm{~B} 3, \mathrm{C} 3$ & M & 74 & ++ & Hipp + BG \\
\hline High NFT 12 & $A D$ & $A 3, B 3, C 3$ & $\mathrm{~F}$ & 88 & ++ & Hipp \\
\hline High NFT 13 & $A D$ & $\mathrm{~A} 3, \mathrm{~B} 3, \mathrm{C} 3$ & M & 79 & ++ & Hipp + BG \\
\hline High NFT 14 & $A D$ & $\mathrm{~A} 3, \mathrm{~B} 3, \mathrm{C} 3$ & $\mathrm{~F}$ & 83 & ++ & Hipp \\
\hline High NFT 15 & AD (early onset) & $\mathrm{A} 3, \mathrm{~B} 3, \mathrm{C} 3$ & M & 69 & ++ & Hipp \\
\hline High NFT 16 & AD (early onset) & $\mathrm{A} 3, \mathrm{~B} 3, \mathrm{C} 3$ & M & 63 & ++ & Hipp \\
\hline High NFT 17 & AD (early onset) & $\mathrm{A} 3, \mathrm{~B} 3, \mathrm{C} 3$ & M & 63 & ++ & Hipp \\
\hline High NFT 18 & AD (early onset) & $A 3, B 3, C 3$ & $\mathrm{~F}$ & 60 & ++ & Hipp \\
\hline High NFT 19 & AD (early onset) & $\mathrm{A} 3, \mathrm{~B} 3, \mathrm{C} 3$ & M & 62 & ++ & Hipp \\
\hline High NFT 20 & AD (early onset) & $A 3, B 3, C 3$ & $\mathrm{~F}$ & 55 & ++ & Hipp \\
\hline High NFT 21 & $A D$ & $\mathrm{~A} 2, \mathrm{~B} 2, \mathrm{C} 2$ & M & 84 & ++ & Hipp \\
\hline Moderate NFT 1 & $A D$ & $A 2, B 3, C 2$ & M & 94 & + & Hipp \\
\hline Moderate NFT 2 & $A D$ & $\mathrm{~A} 3, \mathrm{~B} 3, \mathrm{C} 3$ & $\mathrm{~F}$ & 76 & + & Hipp \\
\hline Moderate NFT 3 & $A D$ & $\mathrm{~A} 3, \mathrm{~B} 3, \mathrm{C} 3$ & M & 69 & + & Hipp \\
\hline Moderate NFT 4 & $A D$ & $\mathrm{~A} 3, \mathrm{~B} 3, \mathrm{C} 3$ & M & 75 & + & Hipp \\
\hline Moderate NFT 5 & $A D$ & $\mathrm{~A} 3, \mathrm{~B} 3, \mathrm{C} 3$ & $\mathrm{~F}$ & 89 & + & Hipp \\
\hline Moderate NFT 6 & $\mathrm{MCl}$ & $\mathrm{A} 1, \mathrm{~B} 2, \mathrm{C} 1$ & $\mathrm{~F}$ & 97 & + & Hipp \\
\hline Moderate NFT 7 & $\mathrm{MCl}$ & $\mathrm{A} 1, \mathrm{~B} 2, \mathrm{C} 1$ & $\mathrm{~F}$ & 85 & + & Hipp \\
\hline Moderate NFT 8 & $\mathrm{MCl}$ & $A 2, B 2, C 2$ & M & 84 & + & Hipp \\
\hline Moderate NFT 9 & $A D$ & $A 2, B 2, C 2$ & $\mathrm{~F}$ & 84 & + & Hipp \\
\hline Moderate NFT 10 & $\mathrm{MCl}$ & $\mathrm{A} 1, \mathrm{~B} 1, \mathrm{CO}$ & $\mathrm{F}$ & 84 & + & Hipp \\
\hline Moderate NFT 11 & $\mathrm{MCl}$ & $\mathrm{A} 1, \mathrm{~B} 1, \mathrm{C} 1$ & M & 79 & + & Hipp \\
\hline Moderate NFT 12 & $\mathrm{MCl}$ & $\mathrm{A} 1, \mathrm{~B} 1, \mathrm{C} 1$ & M & 90 & + & Hipp \\
\hline Moderate NFT 13 & $\mathrm{MCl}$ & $\mathrm{A} 1, \mathrm{~B} 1, \mathrm{C} 1$ & M & 74 & + & Hipp \\
\hline Moderate NFT 14 & $\mathrm{MCl}$ & $\mathrm{A} 1, \mathrm{~B} 1, \mathrm{C} 0$ & M & 95 & + & Hipp \\
\hline Moderate NFT 15 & Normal & $\mathrm{A} 1, \mathrm{~B} 1, \mathrm{C} 0$ & M & 77 & + & Hipp \\
\hline Moderate NFT 16 & $\mathrm{MCl}$ & $\mathrm{AO}, \mathrm{B} 1, \mathrm{CO}$ & M & 88 & + & Hipp \\
\hline Moderate NFT 17 & Vascular Dementia & $\mathrm{A} 1, \mathrm{~B} 1, \mathrm{C} 0$ & $\mathrm{~F}$ & 89 & + & Hipp \\
\hline Moderate NFT 18 & Normal & $\mathrm{A} 1, \mathrm{~B} 1, \mathrm{C} 0$ & M & 69 & + & Hipp \\
\hline Moderate NFT 19 & Normal & $\mathrm{AO}, \mathrm{B} 1, \mathrm{CO}$ & $\mathrm{F}$ & 56 & + & Hipp \\
\hline Moderate NFT 20 & Normal & $\mathrm{AO}, \mathrm{B} 1, \mathrm{CO}$ & $\mathrm{F}$ & 59 & + & Hipp \\
\hline Moderate NFT 21 & Normal & $\mathrm{A} 0, \mathrm{~B} 1, \mathrm{CO}$ & M & 59 & + & Hipp \\
\hline
\end{tabular}


Table 1 Individual case information. Cases were recoded according to their PHF1\% load in hippocampus into their respective NFT score (" $-\leq 0.01 \% ; 0.01 \%<"+$ " $\leq 1 \% ; "++">1 \%)$. Hipp = Hippocampus. BG = Basal Ganglia. TCx=Temporal Cortex. ADRC = Alzheimer's disease related changes (Continued)

\begin{tabular}{|c|c|c|c|c|c|c|}
\hline ID & Diagnosis & $A B C$ score & Sex & Age & NFT score & Region \\
\hline Moderate NFT 22 & Normal & $\mathrm{A} 1, \mathrm{~B} 1, \mathrm{C} 0$ & $\mathrm{~F}$ & 71 & + & Hipp \\
\hline Low/no NFT 1 & Normal & $\mathrm{AO}, \mathrm{BO}, \mathrm{CO}$ & M & 77 & - & Hipp \\
\hline Low/no NFT 2 & Normal & $\mathrm{AO}, \mathrm{BO}, \mathrm{CO}$ & $\mathrm{F}$ & 49 & - & Hipp \\
\hline Low/no NFT 3 & Normal & $\mathrm{A} 1, \mathrm{~B} 1, \mathrm{C} 0$ & M & 63 & - & Hipp \\
\hline Low/no NFT 4 & Normal & $\mathrm{AO}, \mathrm{BO}, \mathrm{CO}$ & $\mathrm{F}$ & 51 & - & Hipp \\
\hline Low/no NFT 5 & Normal & $\mathrm{AO}, \mathrm{BO}, \mathrm{CO}$ & M & 55 & - & Hipp \\
\hline Low/no NFT 6 & Normal & $\mathrm{AO}, \mathrm{BO}, \mathrm{CO}$ & M & 57 & - & Hipp \\
\hline Low/no NFT 7 & Normal & $\mathrm{AO}, \mathrm{BO}, \mathrm{CO}$ & M & 59 & - & Hipp \\
\hline Low/no NFT 8 & Normal & $\mathrm{AO}, \mathrm{BO}, \mathrm{CO}$ & M & 57 & - & Hipp \\
\hline Low/no NFT 9 & Normal & $\mathrm{AO}, \mathrm{BO}, \mathrm{CO}$ & M & 55 & - & Hipp \\
\hline Low/no NFT 10 & Normal & $\mathrm{AO}, \mathrm{BO}, \mathrm{CO}$ & M & 54 & - & Hipp \\
\hline Low/no NFT 11 & Normal & $\mathrm{AO}, \mathrm{BO}, \mathrm{CO}$ & M & 50 & - & Hipp \\
\hline Low/no NFT 12 & Normal & $\mathrm{A} 1, \mathrm{~B} 0, \mathrm{CO}$ & M & 59 & - & Hipp \\
\hline Low/no NFT 13 & Normal & $\mathrm{A} 1, \mathrm{~B} 1, \mathrm{C} 1$ & M & 89 & - & Hipp \\
\hline Low/no NFT 14 & $\mathrm{MCl}$ & $\mathrm{A} 2, \mathrm{~B} 1, \mathrm{C} 1$ & $\mathrm{~F}$ & 89 & - & Hipp \\
\hline Low/no NFT 15 & $\mathrm{MCl}$ & $\mathrm{A} 2, \mathrm{~B} 1, \mathrm{C} 1$ & M & 66 & - & Hipp \\
\hline Down Syndrome 1 & $D S, A D$ & $\mathrm{~A} 3, \mathrm{~B} 3, \mathrm{C} 3$ & $\mathrm{~F}$ & 58 & +++ & Hipp \\
\hline Down Syndrome 2 & $D S, A D$ & $\mathrm{~A} 3, \mathrm{~B} 3, \mathrm{C} 3$ & $\mathrm{~F}$ & 59 & +++ & Hipp \\
\hline Down Syndrome 3 & $D S, A D$ & $\mathrm{~A} 3, \mathrm{~B} 3, \mathrm{C} 3$ & M & 54 & +++ & Hipp \\
\hline Down Syndrome 4 & $D S, A D$ & $\mathrm{~A} 3, \mathrm{~B} 3, \mathrm{C} 3$ & M & 55 & +++ & Hipp \\
\hline Down Syndrome 5 & $D S, A D$ & $\mathrm{~A} 3, \mathrm{~B} 3, \mathrm{C} 3$ & $\mathrm{~F}$ & 54 & +++ & Hipp \\
\hline PART 1 & PART & $\mathrm{AO}, \mathrm{B} 2, \mathrm{CO}$ & M & 75 & + & Hipp \\
\hline PART 2 & PART & $\mathrm{AO}, \mathrm{B} 2, \mathrm{CO}$ & $\mathrm{F}$ & 86 & ++ & Hipp \\
\hline PART 3 & PART & $\mathrm{AO}, \mathrm{B} 2, \mathrm{CO}$ & $\mathrm{F}$ & 92 & + & Hipp \\
\hline PART 4 & PART & $\mathrm{A} 0, \mathrm{~B} 2, \mathrm{CO}$ & M & 94 & + & Hipp \\
\hline PART 5 & PART & $\mathrm{AO}, \mathrm{B} 2, \mathrm{CO}$ & $\mathrm{F}$ & 90 & ++ & Hipp \\
\hline PART6 & PART & $\mathrm{AO}, \mathrm{B} 2, \mathrm{CO}$ & M & 92 & ++ & Hipp \\
\hline CBD 1 & CBD & $\mathrm{n} / \mathrm{a}$ & $\mathrm{F}$ & 73 & +++ & BG \\
\hline CBD 2 & $\mathrm{CBD}$ & $\mathrm{n} / \mathrm{a}$ & M & 75 & +++ & BG \\
\hline CBD 3 & $\mathrm{CBD}$ & $\mathrm{n} / \mathrm{a}$ & M & 79 & ++ & BG \\
\hline CBD 4 & $\mathrm{CBD}$ & $\mathrm{n} / \mathrm{a}$ & $\mathrm{F}$ & 80 & + & BG \\
\hline CBD 5 & $\mathrm{CBD}$ & $\mathrm{n} / \mathrm{a}$ & M & 68 & + & BG \\
\hline PSP 1 & PSP & $\mathrm{n} / \mathrm{a}$ & M & 74 & + & BG \\
\hline PSP 2 & PSP & $\mathrm{n} / \mathrm{a}$ & M & 71 & ++ & BG \\
\hline PSP 3 & PSP & n/a & M & 71 & + & BG \\
\hline PSP 4 & PSP & $\mathrm{n} / \mathrm{a}$ & M & 71 & + & $B G$ \\
\hline PSP 5 & PSP & n/a & $\mathrm{F}$ & 87 & + & BG \\
\hline PiD 1 & Pick's Disease & $\mathrm{n} / \mathrm{a}$ & M & 67 & + & Hipp \\
\hline PiD 2 & Pick's Disease & $\mathrm{n} / \mathrm{a}$ & M & 67 & ++ & Hipp \\
\hline PiD 3 & Pick's Disease & $\mathrm{n} / \mathrm{a}$ & $\mathrm{F}$ & 71 & +++ & Hipp \\
\hline PiD 4 & Picks Disease & $\mathrm{n} / \mathrm{a}$ & $\mathrm{F}$ & 65 & +++ & Hipp \\
\hline HCHWA-D & Dutch amyloidosis & $\mathrm{n} / \mathrm{a}$ & $\mathrm{F}$ & 54 & $\mathrm{n} / \mathrm{a}$ & TCX \\
\hline
\end{tabular}


Table 1 Individual case information. Cases were recoded according to their PHF1\% load in hippocampus into their respective NFT score ("- $\leq$ 0.01\%; 0.01\%< "+" $\leq 1 \% ; "++">1 \%)$. Hipp = Hippocampus. BG = Basal Ganglia. TCx=Temporal Cortex. ADRC = Alzheimer's disease related changes (Continued)

\begin{tabular}{lllllll}
\hline ID & Diagnosis & ABC score & Sex & Age & NFT score & Region \\
\hline FTLD-ADRC 1 & CBD with AD & A0,B1,C0 & M & 72 & n/a & Hipp \\
FTLD-ADRC 2 & PSP with AD & A1,B3,C0 & F & 79 & n/a & Hipp \\
\hline
\end{tabular}

binary threshold that was consistent for all images. Multiple image analyses were performed: (1) staining intensity in SCRN1 positive pixels, (2) SCRN1 intensity in pTau positive neurofibrillary tangles or dystrophic neurites, (3) SCRN1 intensity outside neurofibrillary tangles and dystrophic neurites. For SCRN1 intensity in NFTs and dystrophic neurites, NFTs and dystrophic neurites were identified by thresholding the PHF1 image to identify all pixels positive for pTau labelling. A mask of the area selected in this PHF1 image was then applied to the corresponding SCRN1 stained image. The number of SCRN1 positive pixels and average intensity of SCRN1 in positive pixels was quantified inside and outside NFTs and dystrophic neurites.

In our first analysis examining cases with $\mathrm{AD}$ associated pathology, statistical comparison of SCRN1 intensity inside and outside NFTs or dystrophic neurites in the cortex and in the hippocampus in all groups was performed using a one-way ANOVA with Tukey's multiple comparisons test. Statistical comparison of SCRN1 intensity inside and outside NFTs or dystrophic neurites in other tauopathies (DS, PART, PiD, CBD, PSP) was performed using a two-tailed paired t-test for each group. Representative images for figures (including HCHWA-D) were collected using a Zeiss LSM700 Confocal Microscope. Individual images were collected every $1 \mu \mathrm{m}$ through the depth of the $8 \mu \mathrm{m}$ sections at $20 \mathrm{x}$ magnification and presented images show the maximum projection image. All images of a particular stain were collected using the same confocal settings.

\section{Human tissue homogenization}

Frozen post-mortem frontal cortex tissue from healthy controls $(\mathrm{n}=2)$ and pathologically confirmed AD cases $(n=2)$ were selected from the same cohort described in Table 1. Grey matter was dissected from each tissue sample and flash frozen until use. Frozen cortical tissue $(250 \pm 20 \mathrm{mg})$ was pulverized and dounce homogenized in $5 \mathrm{~mL} / \mathrm{g}(20 \% \mathrm{w} / \mathrm{v})$ of ice-cold homogenization buffer (50 mM HEPES pH 7.0, $250 \mathrm{mM}$ sucrose, $1 \mathrm{mM}$ EDTA, Protease inhibitor cocktail [cOmplete ${ }^{\mathrm{TM}}$ ULTRA Tablets, Mini, EDTA-free; Millipore Sigma; catalog \#5892791001]) using approximately 25 pestle strokes. Protein concentration was determined using Bradford protein assay and homogenates were aliquoted and stored at $-80^{\circ} \mathrm{C}$ until use.

\section{Co-immunoprecipitation}

Immunoprecipitation of SCRN1 was performed using $300 \mu \mathrm{g}$ of human brain homogenate, and $2 \mu \mathrm{g}$ of antiSCRN1 (LSBio; catalog \#LS-C162903) or rabbit IgG isotype control (Thermo Fisher Scientific, catalog \#026102) antibodies. Antibody and brain homogenate were incubated overnight at $4{ }^{\circ} \mathrm{C}$. Immunocomplexes were then incubated with $1.5 \mathrm{mg}$ Dynabeads Protein G magnetic beads (Invitrogen; catalog \#1003D) overnight at $4{ }^{\circ} \mathrm{C}$. Beads were washed four times and IP product was eluted in elution buffer (glycine $\mathrm{pH} 2.8$ ).

\section{Western blot analysis}

Co-IP products and human brain homogenates were analyzed using Western Blot. Samples were mixed in Bolt ${ }^{\text {tw }}$ LDS Sample Buffer (Life Technologies) supplemented with $100 \mathrm{mM}$ 1,4-Dithiothreitol (DTT) and boiled $5 \mathrm{~min}$ at $95^{\circ} \mathrm{C}$. For pTau western blot, samples were processed without DTT or boiling in order to preserve the oligomeric organization of the paired helical filaments. Proteins were resolved on 12-4\% Bis-Tris gels (Life Technologies) and transferred to $0.2 \mu \mathrm{m}$ nitrocellulose membranes (Bio-Rad). Blots were blocked with $5 \%$ milk in TBST for $1 \mathrm{~h}$ and probed with primary antibodies at room temperature for $1 \mathrm{~h}$. Western blot results were visualized using enhanced chemiluminescence (Pierce ECL; Thermo Scientific; \#32106). Signals were captured using ChemiDoc imaging system (Bio-Rad). The following primary antibodies were used (dilutions): anti-pTau PHF1 (1:200; kindly provided by Dr. P.Davies), anti-Tau Phospho (Ser404, rabbit polyclonal, 1:3000; BioLegend; catalog \#SIG-39472), anti-SCRN1 (rabbit polyclonal, 1: 1000; LSBio; catalog \#LS-C162903), anti-SCRN1 (mouse monoclonal, 1:250; LSBio; catalog \#LS-C338451), and anti-GAPDH (1:2000; Cell Signaling; catalog \#97166S). Secondary antibodies were anti-rabbit and anti-mouse horseradish peroxidase-labeled antibodies (both 1:3000; GE Healthcare).

\section{Results}

Secernin-1 distribution in the brain throughout the progression of $A D$

In order to determine the physiological localization of SCRN1 and map the accumulation of SCRN1 throughout the progression of $\mathrm{AD}$, we used immunohistochemistry to compare SCRN1 distribution in cases with high 
AD-associated NFT pathology ( $n=21$ cases), moderate AD-associated NFT pathology $(n=22)$ and in cognitively normal controls with little or no NFT pathology $(\mathrm{n}=$ 15). Cases were included in a specific group according to their NFT load as described in Table 1. SCRN1 was consistently observed in the neuronal cytoplasm in control subjects, confirming that it is a physiological neuronal protein (Fig. 1a-f). The amount of basal SCRN1 neuronal staining in neurons not containing NFTs was similar between the three groups (Fig. 3c, d). There also appeared to be regional differences of basal SCRN1 immunoreactivity: Comparison of SCRN1 in CA1, CA2, CA3 and cortical regions showed comparatively higher SCRN1 staining intensity in CA3, entorhinal cortex and temporal cortex, and lower levels in CA2 and CA1 (Fig. 1). These basal regional differences were observed in all cases regardless of pathology. There was also consistent and bright staining of SCRN1 in the choroid plexus, suggesting that there may be potential secretion, transport or clearance of SCRN1 into or out of the CSF.

A striking pattern of SCRN1 staining was observed in the cases with AD-associated neuropathology. Particularly bright SCRN1 staining was observed in neurofibrillary tangles and in dystrophic neurites present in neuritic plaques (Fig. 2). To determine if there was a particular colocalization of SCRN1 with specific pTau species, we performed double immunohistochemistry of SCRN1 with the antibodies PHF1 (raised against pS404/pS396), AT8 (raised against pSer202/pThr205) and MC1 (conformation-dependent antibody; commonly used as a marker of early-stage tangles). SCRN1 showed consistent colocalization with all three markers, with a striking and obvious accumulation in tangle-bearing neurons and in dystrophic neurites regardless of the pTau species present (Fig. 2). We confirmed that SCRN1 was associated with $A \beta$ plaques, however, the colocalization was limited to the plaque-associated dystrophic neurites rather than the $A \beta$ itself (Fig. 2a-c).

In order to determine the timing and extent of SCRN1 accumulation in NFTs we quantified and compared the average staining intensity of SCRN1 inside and outside PHF1-positive NFTs in the cortex and hippocampus in cases with high, moderate and low NFT pathology (Fig. 3a). We found significantly more SCRN1 in NFTs in comparison to surrounding neurons in all cases where NFTs were present (Fig. 3c, d). Importantly, accumulation of SCRN1
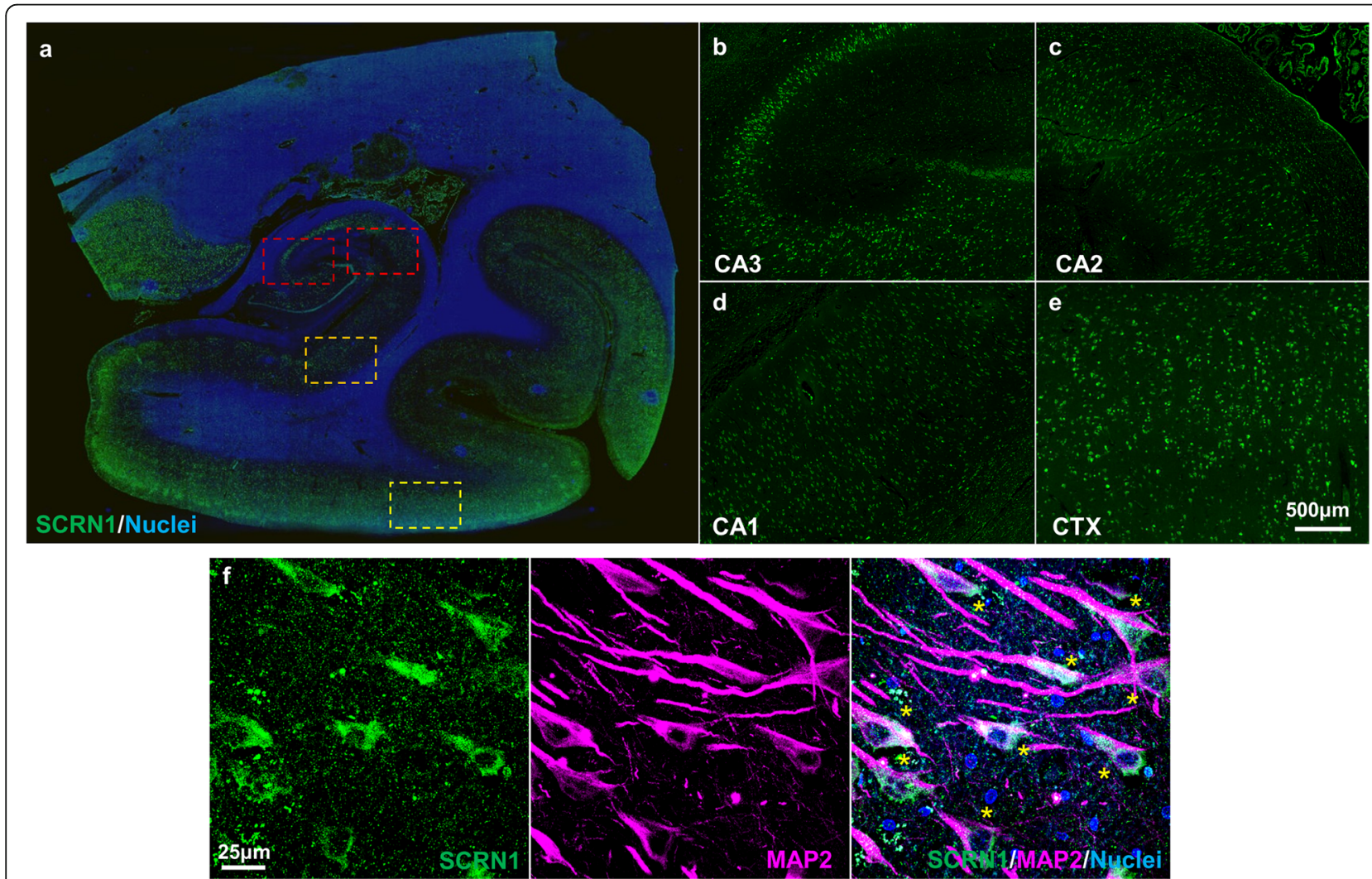

Fig. 1 Secernin-1 distribution in the hippocampal section in a cognitively normal subject with no NFTs. a Whole-slide fluorescent scan of the hippocampus with a-SCRN1 antibody (green). Boxed regions shown in higher magnification images of CA3 (dark red, b), CA2 (red, c), CA1 (orange, $\mathbf{d}$ ) and adjacent temporal cortex (yellow, e). f Double immunohistochemistry of SCRN1 with a-MAP2 antibody showing SCRN1 physiological expression in neurons (asterix) in a cognitively normal subject 


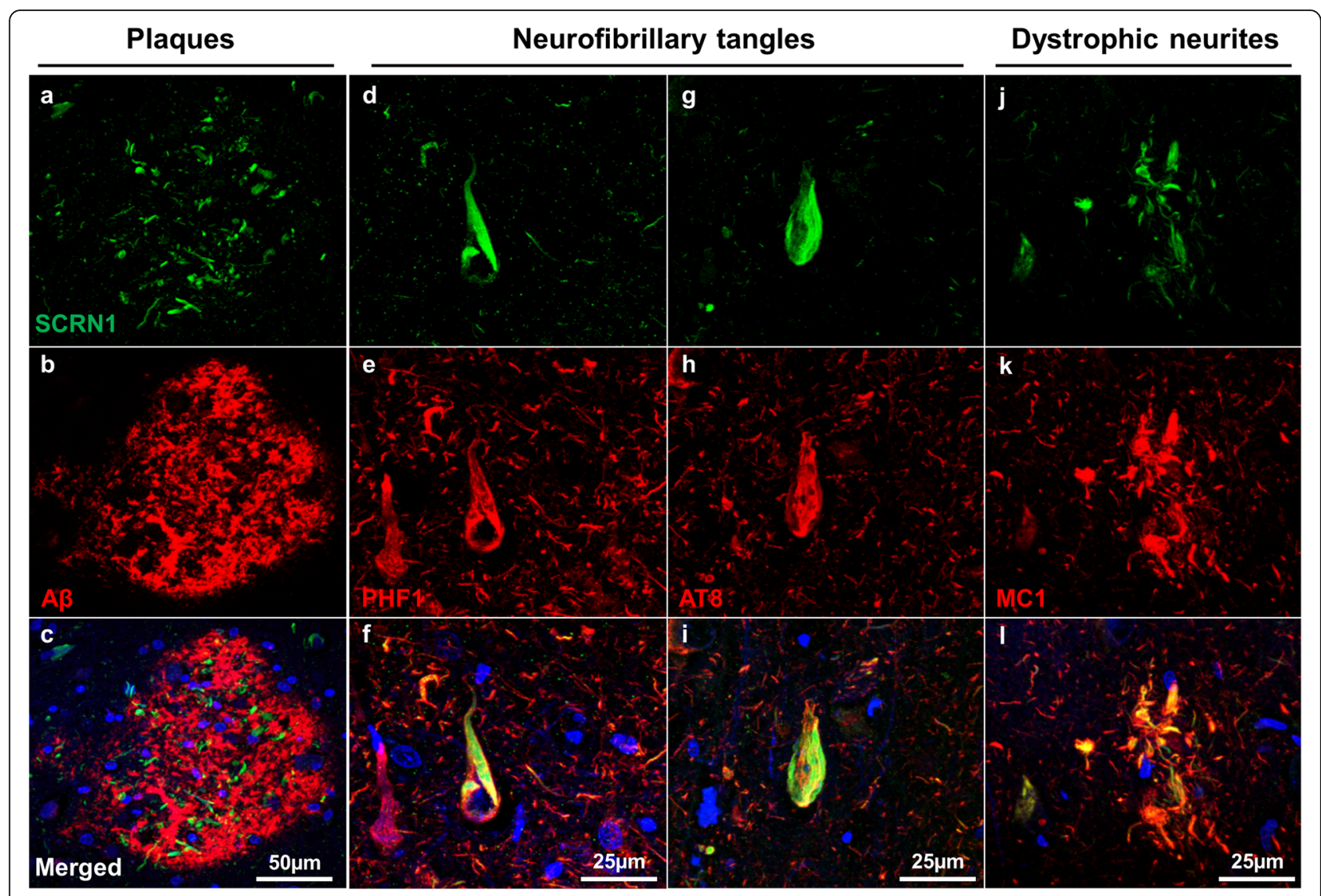

Fig. 2 Secernin-1 accumulation in NFTs and dystrophic neurites in AD. Sections were immunostained with a-SCRN1 and 4G8/6E10 (aß, a-c) or the pTau markers PHF1 (d-f), AT8 ( $\mathbf{g}-\mathbf{i})$ and MC1 (j-l)

was consistently observed in the small number of earlystage NFTs present in the moderate NFT and low/no NFT group (Fig. 3d). This observation, coupled with the colocalization of SCRN1 with the early stage NFT marker MC1, suggests that SCRN1 accumulates early in the formation of NFTs and may therefore have an early role in tangle pathology.

\section{Secernin-1 does not accumulate in pTau-positive lesions} in 3R-tau and 4R-tau specific tauopathies

To address whether SCRN1 also accumulated in NFTs and dystrophic neurites in other tauopathies, we performed double fluorescent immunohistochemistry on basal ganglia or hippocampus sections from cases of CBD $(n=5)$, PSP $(n=5)$ and PiD $(n=4)$ and compared it to those of $\mathrm{AD}$ cases $(n=4$ of hippocampus, $\mathrm{n}=4$ of basal ganglia). These tauopathies are known as 4R-Tau (CBD, PSP) or 3R-Tau specific (PiD) tauopathies as the pTau positive lesions present in these diseases exclusively contain either the 4R-Tau or 3R-Tau isoforms. For each disease, we also stained sections with RD3 and RD4 antibodies to confirm the exclusive presence of $4 \mathrm{R}$ Tau in the CBD and PSP cases and of 3R-Tau isoforms in the PiD cases (Fig. 5d-f, g-i). Presence of both 3R and 4R-Tau isoforms was confirmed in the AD cases in both the hippocampus and basal ganglia. In the 4R-Tau specific tauopathies, we observed extensive PHF1 positive staining consistent with the presence of astrocytic plaques (CBD), tufted astrocytes (PSP), pre-tangles, and dystrophic neurites (Fig. 4a) in the basal ganglia. PHF1 pathology in PiD was consistent with Pick's bodies present in the dentate gyrus, CA regions or entorhinal/ transentorhinal region of the cortex (Fig. 4a). Surprisingly, SCRN1 did not accumulate inside any of these pTau immunoreactive lesions in CBD, PSP or PiD (Fig. 4a, Fig. 5d-f, g-i). Quantification of SCRN1 intensity inside and outside PHF1-positive lesions confirmed our findings (Fig. 4d, e), showing that SCRN1 accumulation is a feature unique to NFTs in AD where both 3R-Tau and 4R-Tau isoforms are present and that this occurs across multiple brain regions (hippocampus, cortex and basal ganglia examined in this study). Interestingly, we also observed that SCRN1 consistently colocalized with both 3R or 4R-Tau in NFTs in AD, therefore providing evidence that SCRN1 did not preferentially associate with either 3R or 4R-Tau isoforms (Fig. 5a-c). 


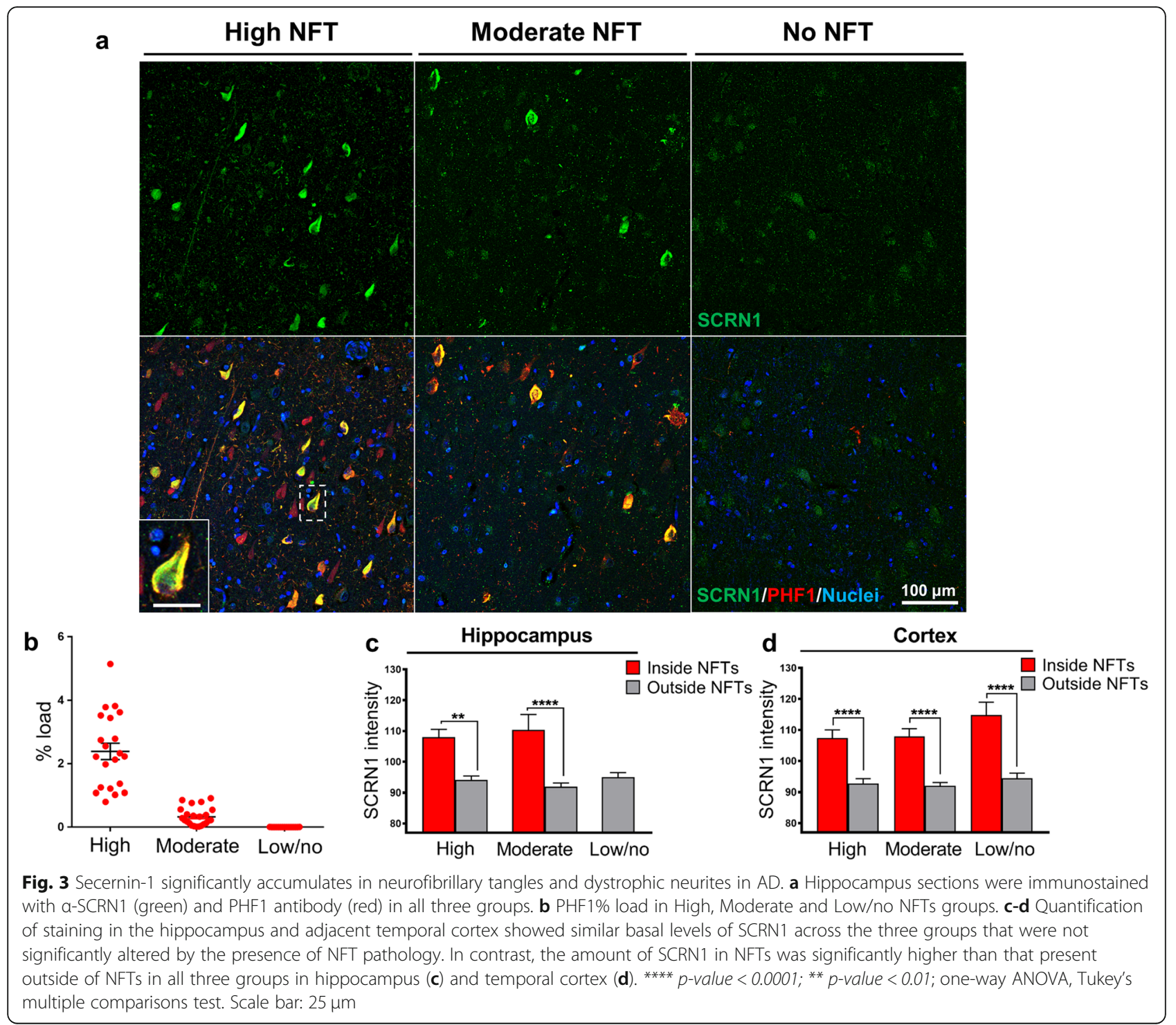

Secernin-1 accumulates in AD-associated NFTs in mixed pathology cases with both FTLD-tau and AD-related neuropathology

We also examined SCRN1 distribution in two rare cases with mixed pathology: the first subject primarily had CBD-associated neuropathology characterized by astrocytic plaques and pre-tangles, but also had a small number of AD-associated NFTs in the entorhinal cortex and hippocampus. Interestingly, only PHF1 positive lesions with morphology consistent with AD-associated NFTs showed SCRN1 accumulation in this case (Fig. 6g). Given the specificity of $4 \mathrm{R}$ tau in lesions present in CBD, we then performed $3 R$ and $4 R$ specific immunohistochemistry on this case of mixed pathology to see if SCRN1 was preferentially present in lesions containing 3R tau. Indeed, this analysis showed striking accumulation of SCRN1 in NFTs containing both 3R and 4R tau
(Fig. 6h, i), but not with pre-tangles containing only $4 \mathrm{R}$ tau (Fig. 6e). Interestingly, SCRN1 was predominantly present in dystrophic neurites that were immunoreactive for PHF1-positive pTau or 3R tau (Fig. 6g, i) in comparison to those that contained $4 \mathrm{R}$ tau (Fig. 6h). The second case of mixed pathology that we examined presented with pTau immunoreactive tufted astrocytes that are commonly observed in PSP, and a small number of thorn-shaped astrocytes that are commonly found in aging-related tau astrogliopathy (ARTAG) [25, 47, 48]. There was also evidence of pre-tangles, as well as some AD-associated NFTs in the hippocampus and adjacent entorhinal cortex. In this case, the most predominant SCRN1 accumulation was again observed in ADassociated NFTs (Fig. 7a, b) and there was no evidence of SCRN1 in tufted astrocytes (Fig. 7c, d). Unexpectedly, we did observe a small amount of SCRN1 accumulation 


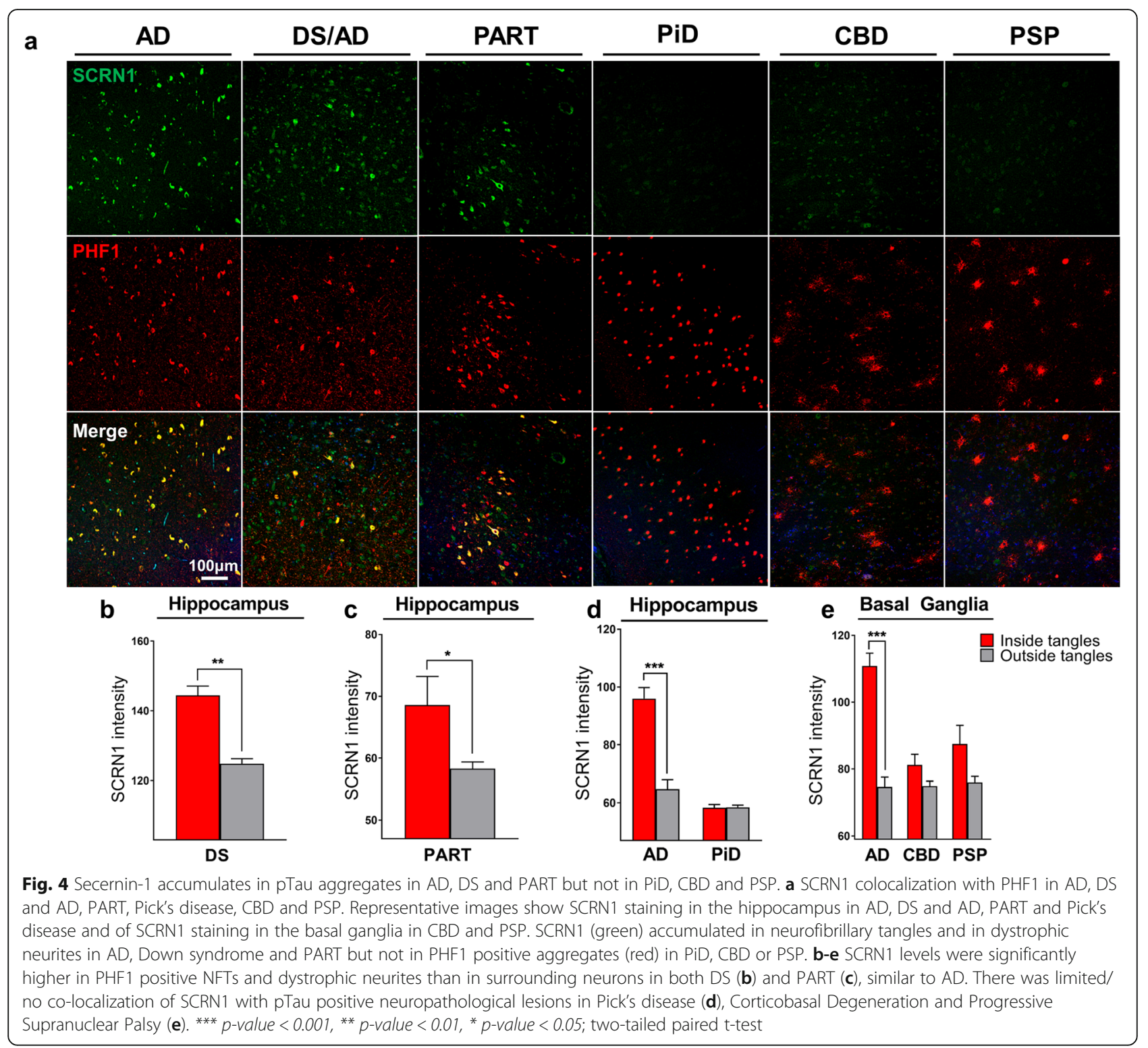

inside thorn-shaped astrocytes (Fig. 7e, f). However in contrast to the widespread SCRN1 colocalization in NFTs, the presence of SCRN1 in thorn-shaped astrocytes was limited to a small proportion of the pTau aggregate that was primarily adjacent to the nucleus (Fig. 7a, b; Fig. 2; Fig. 4a).

\section{Secernin-1 accumulates in NFTs present in cases of aged Down syndrome and in PART}

SCRN1 expression was also examined in cases of DS with AD as well as cases of PART. Individuals with DS all develop AD pathology at an early age [38, 52]. The triplication of the amyloid precursor protein (APP) gene on chromosome 21 results in a progressive accumulation of $A \beta$ starting in early life, such that, by middle age, all people with DS will develop advanced AD pathology.
PART is characterized by tau aggregation confined to the entorhinal cortex and hippocampus with no or minimal $A \beta$ deposition [10]. We found that the SCRN1 distribution in DS with $\mathrm{AD}$ and PART was similar to that observed in AD; SCRN1 accumulated in NFTs in both types of disease and was present in the dystrophic neurites present in neuritic plaques in DS with AD (Fig. 4a, $\mathrm{b}, \mathrm{c})$. These findings suggest that there is a similar mechanism in place for SCRN1 accumulation in AD, DS with AD pathology, and PART.

\section{Secernin-1 in HCHWA-D}

We also analyzed SCRN1 distribution in the brain in HCHWA-D, which is a rare autosomal dominant disorder caused by an APP 693 mutation that clinically leads to recurrent hemorrhagic strokes and dementia [4]. 


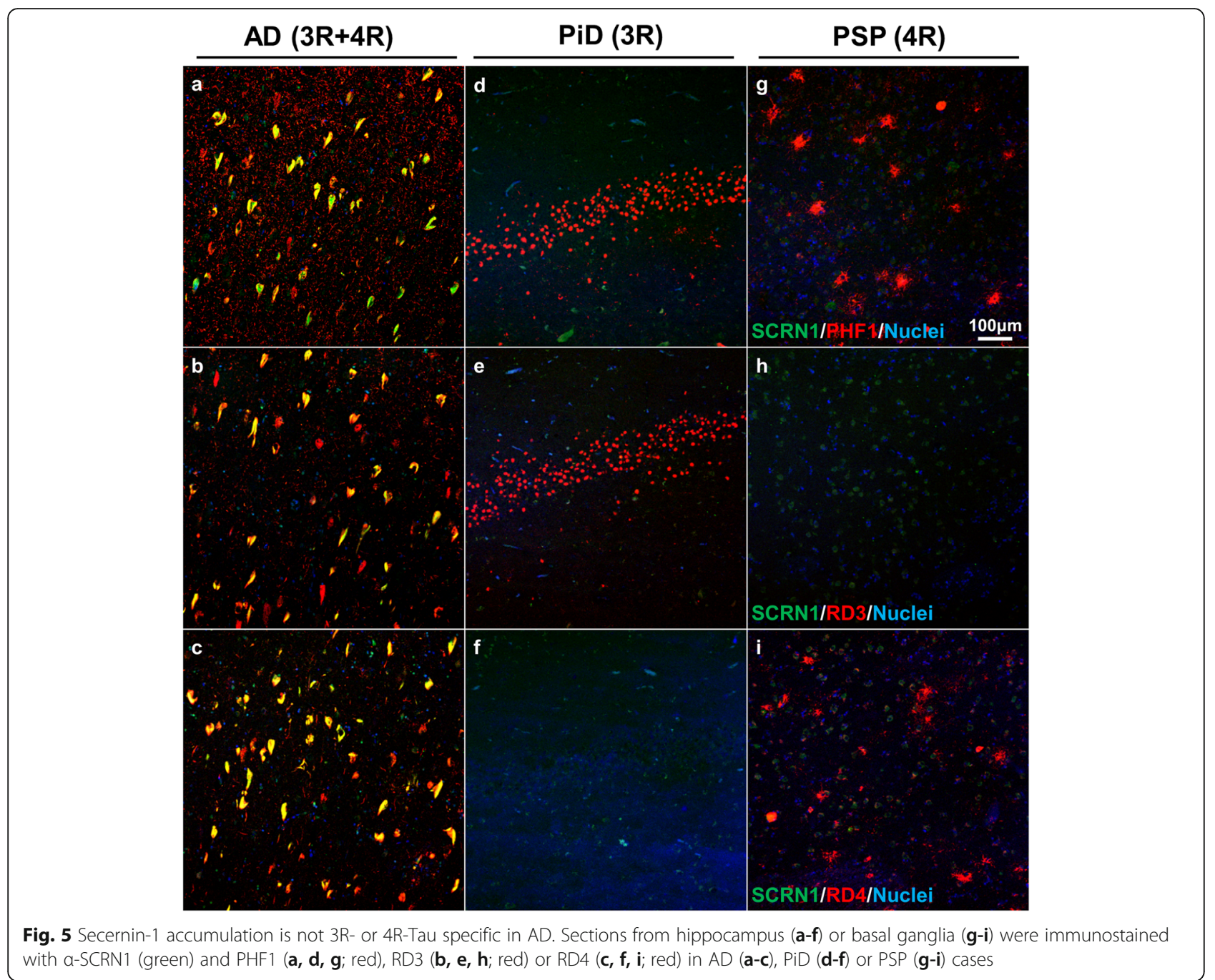

The disease is pathologically characterized by CAA and amyloid plaques, without presence of neurofibrillary pathology [4]. In this case, we did not observe any pathologic accumulation of SCRN1, either in vessels or in plaques (Fig. 8).

\section{Secernin-1 interacts with PHF-tau}

The robust colocalization of SCRN1 and pTau observed using immunohistochemistry implies that there may be a direct interaction between SCRN1 and pTau. To test whether this was the case, we performed co-IP of pTau and SCRN1 using fresh-frozen human prefrontal cortex tissue. Immunoprecipitation of SCRN1 from human AD brain homogenates resulted in the co-IP of pTau, implying that SCRN1 interacts with PHF-Tau in vivo (Fig. 9). There was no evidence of any interaction of SCRN1 and pTau in control samples ( $n=2$; Fig. 9).

\section{Discussion}

Here, we present a comprehensive neuropathological study showing that SCRN1 is a novel protein that accumulates specifically in AD, DS and PART, which are $3 R / 4 R$ tauopathies, but not in other tauopathies that are either $3 R$ or $4 R$ tauopathies. To our knowledge, this is the first example of a protein that distinguishes tau aggregates in different tauopathies. We found that SCRN1 strongly colocalized with NFTs and dystrophic neurites in all brain regions that we examined using $\mathrm{AD}$ brain tissue and found SCRN1 to directly interact with phosphorylated tau in $\mathrm{AD}$, a finding that was recently confirmed in an additional study. This study showed significant interaction between SCRN1 and tau in AD, but only minimal interaction in controls, supporting an $\mathrm{AD}$ specific interaction between these two proteins [40]. Importantly, SCRN1 was observed in NFTs and dystrophic neurites in preclinical stages of the AD process, suggesting that it could be a key factor associated with the development of NFTs in AD.

The absence of SCRN1 accumulation in pTau lesions present in the 4R-specific tauopathies CBD and PSP raised the possibility that SCRN1 preferentially 


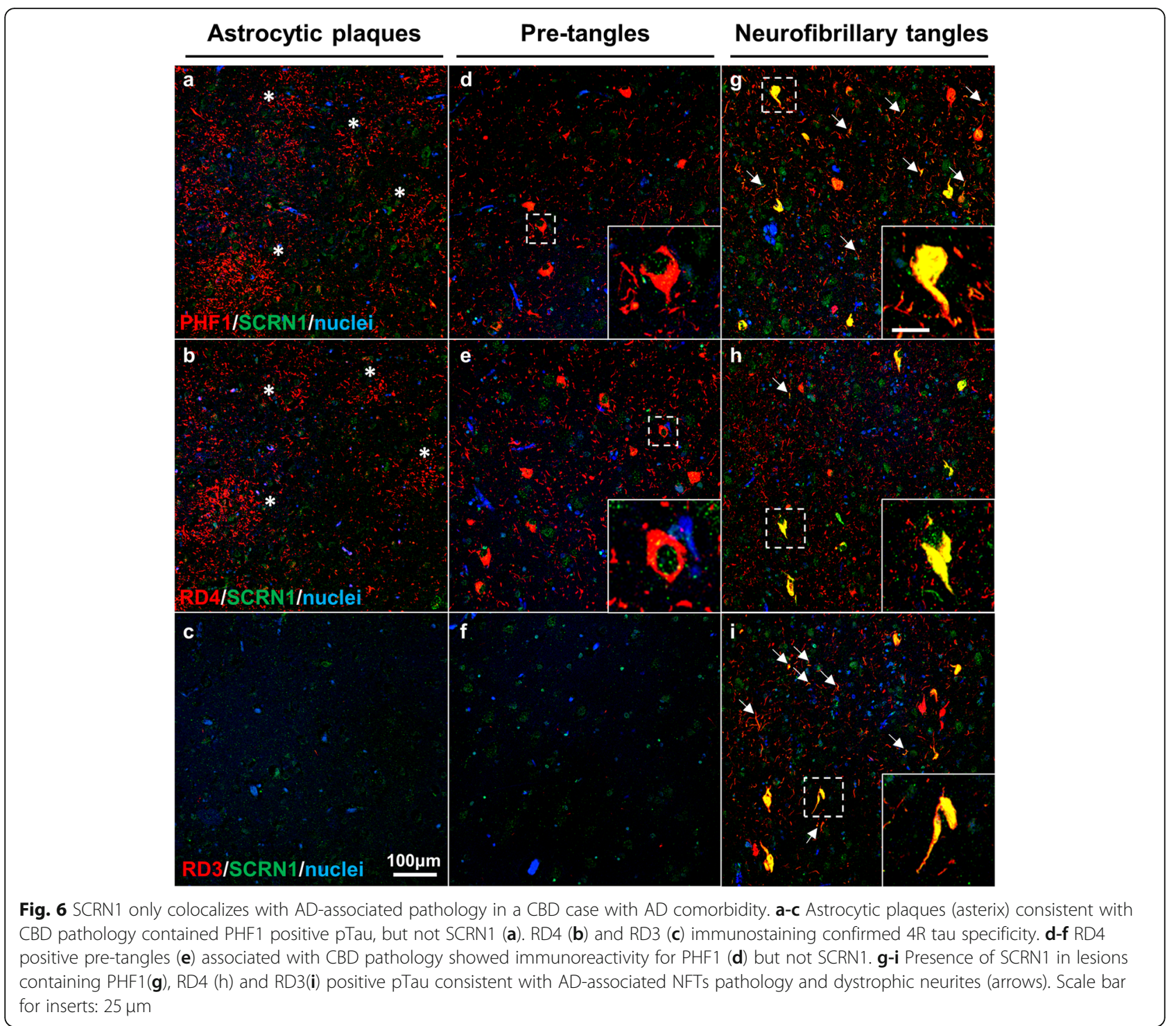

colocalizes with 3R-tau isoforms, however the lack of SCRN1 accumulation in the 3R-tau specific PiD showed that this was not the case. Adding to the complexity of the relationship between SCRN1 and pTau was the finding that SCRN1 exclusively colocalized with pTau aggregates with NFT morphology and not those aggregates with CBD and PSP-associated morphology in mixed pathology cases. Interestingly, very limited presence of SCRN1 was observed in thorn-shaped astrocytes in one of the mixed pathology cases. Thorn-shaped astrocytes are commonly present in ARTAG, a specific neuropathological condition defined by the existence of pTaubearing astrocytes in the brain of old-aged individuals $[25,47,48]$. This finding shows that astrocytes have the ability to accumulate SCRN1, suggesting that the absence of SCRN1 in tufted astrocytes or astrocytic plaques is not due to SCRN1 neuronal specificity.
SCRN1 colocalization was also evident in cases of DS with $\mathrm{AD}$ and in PART. Importantly, neurofibrillary tangles in DS and PART also contain typical AD-like PHFs that consist of both $3 R$ and $4 R$ tau isoforms [10, 18, 37]. Recent cryo-electron microscopy studies of AD NFTs show that the tau aggregates contain a distinct $\mathrm{C}$-shaped curve with the $3 \mathrm{R}$ and $4 \mathrm{R}$ tau included in the core of the fibrils $[19,27]$. Hence the $3 R / 4 R$ tau aggregates in $A D$ have a conformation that is distinct from tau species containing only $3 \mathrm{R}$ or $4 \mathrm{R}$ tau. This was supported by the recent finding that pTau aggregates in $\mathrm{AD}, \mathrm{PiD}$ and CBD are characterized by different tau molecular structures with different conformations [21, 22, 29, 76]. Together, these findings suggest that the combination of both $3 R$ and $4 R$ tau isoforms in pTau aggregates in AD, DS with AD, and PART could result in the generation of a common tau conformation, or tau "strain", across 

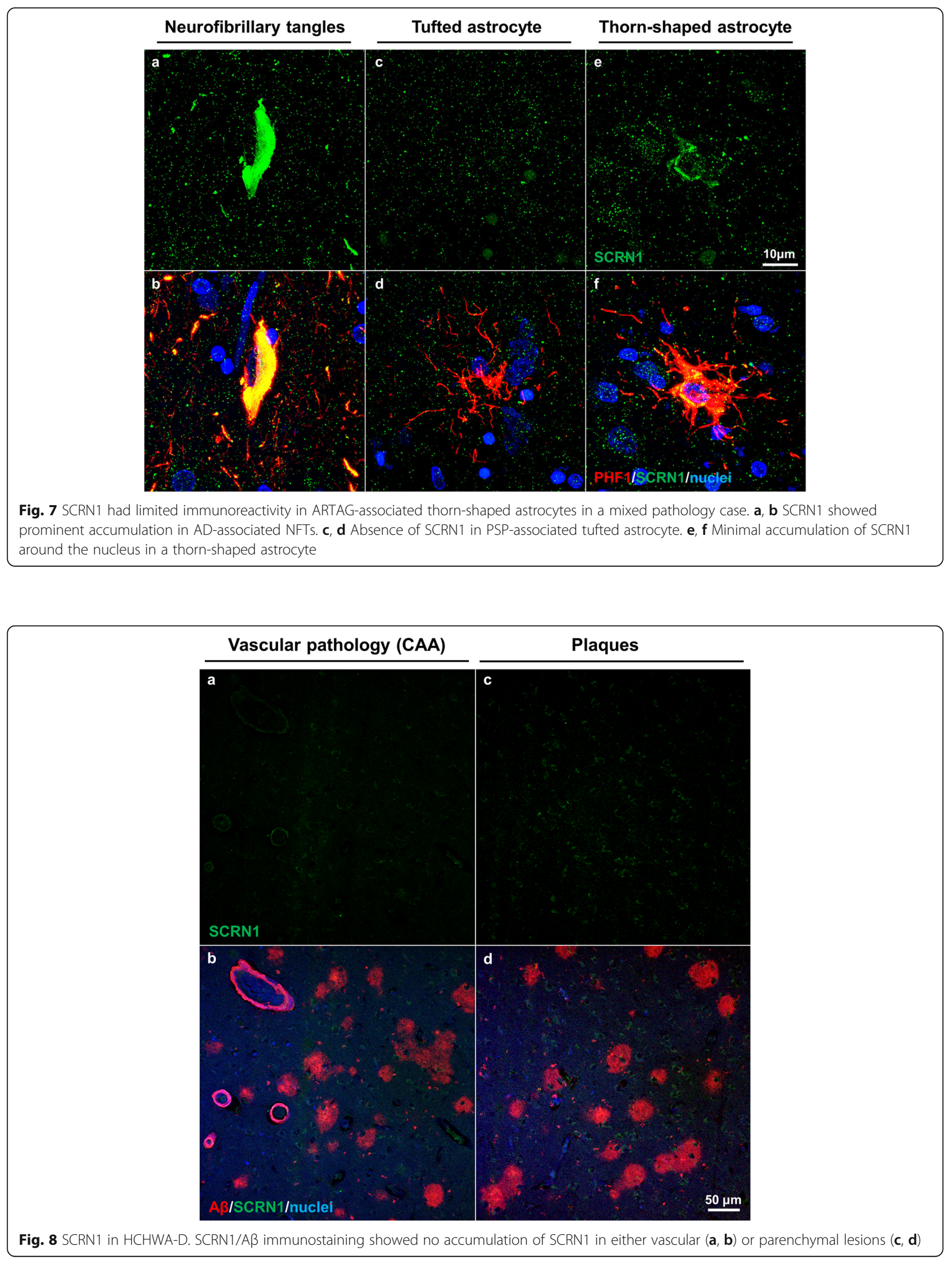


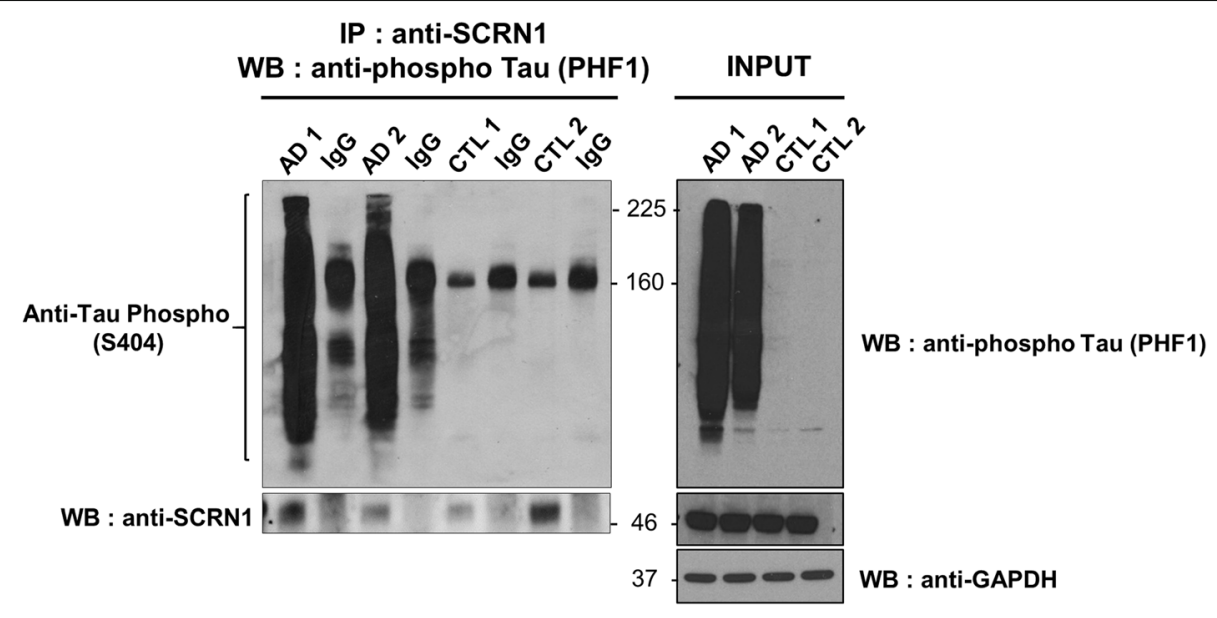

Fig. 9 SCRN1 binds to phosphorylated tau in AD cortex tissue. Co-IP was performed on fresh frozen frontal cortex tissue from $n=2 A D$ and $n=2$ cognitively normal samples. IP was performed using anti-SCRN1 or rabbit IgG isotype control. SCRN1 pulled down phosphorylated tau in AD cortex samples, but not control cortex samples. Fiftee microgram protein per sample from total homogenate were loaded for inputs. Loading control: GAPDH

these diseases that is then capable of interaction with the same protein co-factors, such as SCRN1. Logically, the different conformation of tau aggregates in other primary tauopathies could prevent interaction with SCRN1. Structure-based inhibitors of tau aggregation are now being developed [64]. Hence targeting the SCRN1/pTau interaction may be a novel therapeutic strategy.

An alternative possibility is that the interaction between SCRN1 and pTau may require the presence of both $A \beta$ and tau. This is supported by the finding that SCRN1 accumulation is present in $\mathrm{AD}$, DS where cooccurrence of $A \beta$ and Tau is observed, while it is not present in tau-only dementias (PSP, CBD, PiD) or in HCHWA-D that only has accumulation of $A \beta$. The interplay between $A \beta$ and tau is a crucial factor in $A D$ pathogenesis. It has been suggested that this interplay occurs intraneuronally $[8,36,57,58]$, where the interaction with SCRN1 also occurs. One could speculate that $A \beta$ may influence SCRN1 production or activation and therefore influence the subsequent interaction with pTau. Indeed, $A \beta$ is known to stimulate many pathogenic pathways that ultimately influence tau pathology $[7,45,68,69,75]$. Therefore, it is possible that the interaction between SCRN1 and pTau is mediated by $A \beta$ in a similar way. However, the abundant presence of SCRN1 in PART does not fit with this hypothesis. Whether PART represents an early stage of $\mathrm{AD}$ with very low levels of $A \beta$ or is a distinct pathological entity is still a matter of debate $[10,18]$. In our study, we did not find a correlation between the extent of $A \beta$ pathology and accumulation of SCRN1, but rather observed a consistent accumulation of SCRN1 in NFTs in PART. Together, our results suggest that NFTs in PART resemble those present in early stage $\mathrm{AD}$ pathology.
The mechanistic involvement of SCRN1 in pTau aggregation and NFT formation is unknown. Further studies are ongoing to determine why SCRN1 is present in NFTs. Domain prediction analysis shows that SCRN1 does not contain a kinase domain, implying that it is unlikely to be involved in the phosphorylation of tau. Interestingly, SCRN1 does contain a conserved 142 aminoacid domain present in members of the Peptidase c69 family. These dipeptidases are cysteine-dependent peptidases that specifically hydrolyze X-Pro motifs [63]. A large number of proteases that cleave tau, such as calpains $[24,61]$, cathepsins $[41,61,71]$, caspases $[28,34$, $61,78]$ and the lysosomal protease Asparagine endopeptidase (AEP) [61, 77] are also cysteine proteases that are present in intracellular NFTs in AD, similar to SCRN1. The cleavage of tau has been suggested to seed tau aggregation [72]. Therefore, it would be very interesting for future studies to determine if SCRN1 is possibly a novel protease that is involved in pTau proteolysis or degradation.

This study is an example of the powerful and informative nature of unbiased localized proteomics studies, which permit the discovery of novel disease related proteins present within neuropathological features through unbiased analysis [12-15]. It is important to recognize that SCRN1 was only brought to our attention as a novel $\mathrm{AD}$ associated protein because of the localized nature of our original proteomics study that identified SCRN1 in dystrophic neurites in amyloid plaques [12]. In contrast, previous transcriptomics and proteomics studies using bulk tissue homogenates did not find it to be one of the top proteins altered in $\mathrm{AD}[1,33,44,53,60,65]$. This supports our Western blot results that showed no difference in the amount of SCRN1 in AD and controls in 
total brain homogenate. The obvious striking accumulation of SCRN1that we observed using IHC shows that localized proteomics studies are necessary and that pathologically important protein differences may be missed in studies of bulk tissue. This is additionally supported by the recent report that examined single-cell transcriptomic changes that found SCRN1 mRNA to be increased in neurons in AD, but not in other cell types [54]. This suggests that the accumulation of SCRN1 in NFTs may be a result of increased localized production of SCRN1 in neurons in AD. Future studies using in situ hybridization on human $\mathrm{AD}$ brain sections will help confirm if this increased mRNA production is localized inside NFT containing neurons in AD.

In conclusion, we have shown that the novel protein SCRN1 is associated with neurofibrillary tangles in AD, DS and PART but not in other tauopathies. Future studies both in vitro and in vivo are currently being performed to determine the physiological and pathological function of SCRN1 in the brain, as well as to determine the consequences of its interaction with pTau. Together, our results suggest that SCRN1 is a novel protein that is involved in the pathogenesis of AD.

\section{Supplementary information}

Supplementary information accompanies this paper at https://doi.org/10. 1186/s40478-019-0848-6.

Additional file 1: Figure S1. Confirmation of a-SCRN1antibody specificity. a Western Blot analyses of fresh frozen frontal cortex tissue from $n=$ $2 \mathrm{AD}$ and $n=2$ cognitively normal samples with PHF1 (pTau ser396/ ser404) and two different a-SCRN1 antibodies specifically labelling the 46KDa full-length SCRN1. Immunoblot showed one specific band for SCRN1 and similar SCRN1 levels in AD and cognitively normal samples. Fifteen micrograms protein per sample from total homogenate were loaded. GAPDH was used as loading control. b Absorption assay showing the lack of SCRN1 staining after pre-absorption with human recombinant SCRN1 protein. -ve: negative control (no primary antibody).

\section{Authors' contributions}

GP performed experiments and wrote the paper. SM and SD performed experiments. GH, MCP and TW provided tissue and provided data analysis. TW and ED planned the experiments and wrote the paper. All authors read and approved the final manuscript.

\section{Funding}

This study was supported by funding from the Philippe Chatrier Foundation to GP, the Bluesand Foundation and Dementia Australia to ED; as well as, $\mathrm{NIH}$ grants (to TW) AG008051 and AG058267. The longitudinal brain donor programs were funded by the NHMRC and GMH is a NHMRC Senior Principal Research Fellow (1079679). We thank Prof. Charles Duyckaerts for his helpful suggestions during the preparation of this manuscript.

\section{Availability of data and materials}

The data and material used in this study is available upon request.

\section{Ethics approval and consent to participate}

All cases used in this study were from ethically-approved longitudinally assessed regional brain donor programs on neurodegenerative diseases. All procedures were performed under protocols approved by the New York University Alzheimer Disease Center, NY, the South Eastern Sydney and Illawarra
Local Health District and the Universities of New South Wales and Sydney, Australia.

\section{Consent for publication}

In all cases, written informed consent for research and publication was obtained from the patient or legal guardian, and the material used had appropriate ethical approval for use in this project. All patients' data and samples were coded and handled according to $\mathrm{NIH}$ and NHMRC guidelines to protect patients' identities.

\section{Competing interests}

The authors declare that they have no competing interests.

\section{Author details}

${ }^{1}$ Center for Cognitive Neurology and Department of Neurology, New York University School of Medicine, 435 East 30th Street, Rm 1017, New York, NY 10016, USA. ${ }^{2}$ Departments of Pathology and Psychiatry, New York University School of Medicine, 435 East 30th Street, Rm 1017, New York, NY 10016, USA. ${ }^{3}$ Brain \& Mind Centre and Central Clinical School, Faculty of Medicine and Health, University of Sydney, 94 Mallett Street, Camperdown, NSW 2050, Australia. Institut du Cerveau et de la Moelle épinière, CNRS UMR7225, INSERM U1127, UPMC, Hôpital de la Pitié-Salpêtrière, 47 Bd de l'Hôpital, Paris, France.

Received: 11 November 2019 Accepted: 14 November 2019 Published online: 03 December 2019

\section{References}

1. Annese A et al (2018) Whole transcriptome profiling of late-onset Alzheimer's disease patients provides insights into the molecular changes involved in the disease. Sci Rep 8(1):4282

2. Ayyadevara $\mathrm{S}$ et al (2016) Proteins that mediate protein aggregation and cytotoxicity distinguish Alzheimer's hippocampus from normal controls. Aging Cell 15(5):924-939

3. Bancher C et al (1989) Accumulation of abnormally phosphorylated tau precedes the formation of neurofibrillary tangles in Alzheimer's disease. Brain Res 477(1-2):90-99

4. Bornebroek M et al (2003) Hereditary cerebral hemorrhage with amyloidosis Dutch type (AbetaPP 693): decreased plasma amyloid-beta 42 concentration. Neurobiol Dis 14(3):619-623

5. Buee L, Delacourte A (1999) Comparative biochemistry of tau in progressive supranuclear palsy, corticobasal degeneration, FTDP-17 and Pick's disease. Brain Pathol 9(4):681-693

6. Buee $L$ et al (2000) Tau protein isoforms, phosphorylation and role in neurodegenerative disorders. Brain Res Brain Res Rev 33(1):95-130

7. Bulbarelli A et al (2009) TrkA pathway activation induced by amyloid-beta (Abeta). Mol Cell Neurosci 40(3):365-373

8. Casas C et al (2004) Massive CA1/2 neuronal loss with intraneuronal and Nterminal truncated Abeta42 accumulation in a novel Alzheimer transgenic model. Am J Pathol 165(4):1289-1300

9. Chang SH et al (2013) Proteomic profiling of brain cortex tissues in a tau transgenic mouse model of Alzheimer's disease. Biochem Biophys Res Commun 430(2):670-675

10. Crary JF et al (2014) Primary age-related tauopathy (PART): a common pathology associated with human aging. Acta Neuropathol 128(6):755-766

11. Drummond E, Wisniewski T (2017) The use of localized proteomics to identify the drivers of Alzheimer's disease pathogenesis. Neural Regen Res 12(6):912-913

12. Drummond E et al (2017) Proteomic differences in amyloid plaques in rapidly progressive and sporadic Alzheimer's disease. Acta Neuropathol 133(6):933-954

13. Drummond $E$ et al (2018) Isolation of amyloid plaques and neurofibrillary tangles from archived Alzheimer's disease tissue using laser-capture microdissection for downstream proteomics. Methods Mol Biol 1723:319-334

14. Drummond E et al (2018) Potential novel approaches to understand the pathogenesis and treat Alzheimer's disease. J Alzheimers Dis 64(s1): S299-s312

15. Drummond ES et al (2015) Proteomic analysis of neurons microdissected from formalin-fixed, paraffin-embedded Alzheimer's disease brain tissue. Sci Rep 5:15456 
16. Dujardin S et al (2018) Different tau species lead to heterogeneous tau pathology propagation and misfolding. Acta Neuropathol Commun 6(1):132

17. Duyckaerts C, Delatour B, Potier MC (2009) Classification and basic pathology of Alzheimer disease. Acta Neuropathol 118(1):5-36

18. Duyckaerts $C$ et al (2015) PART is part of Alzheimer disease. Acta Neuropathol 129(5):749-756

19. Eisenberg DS, Sawaya MR (2017) Neurodegeneration: taming tangled tau. Nature 547(7662):170-171

20. Fagerberg $L$ et al (2014) Analysis of the human tissue-specific expression by genome-wide integration of transcriptomics and antibody-based proteomics. Mol Cell Proteomics 13(2):397-406

21. Falcon B et al (2018) Structures of filaments from Pick's disease reveal a novel tau protein fold. Nature 561(7721):137-140

22. Falcon B et al (2018) Tau filaments from multiple cases of sporadic and inherited Alzheimer's disease adopt a common fold. Acta Neuropathol 136(5):699-708

23. Feany MB, Dickson DW (1996) Neurodegenerative disorders with extensive tau pathology: a comparative study and review. Ann Neurol 40(2):139-148

24. Ferreira A, Bigio EH (2011) Calpain-mediated tau cleavage: a mechanism leading to neurodegeneration shared by multiple tauopathies. Mol Med 17(7-8):676-685

25. Ferrer I et al (2018) Aging-related tau astrogliopathy (ARTAG): not only tau phosphorylation in astrocytes. Brain Pathol 28(6):965-985

26. Finnegan $S$ et al (2010) Proteomic profiling of the retinal dysplasia and degeneration chick retina. Mol Vis 16:7-17

27. Fitzpatrick AWP et al (2017) Cryo-EM structures of tau filaments from Alzheimer's disease. Nature 547(7662):185-190

28. Gamblin TC et al (2003) Caspase cleavage of tau: linking amyloid and neurofibrillary tangles in Alzheimer's disease. Proc Natl Acad Sci U S A 100(17):10032-10037

29. Goedert M (2018) Tau filaments in neurodegenerative diseases. FEBS Lett 592(14):2383-2391

30. Goedert M et al (1992) Tau proteins of Alzheimer paired helical filaments: abnormal phosphorylation of all six brain isoforms. Neuron 8(1):159-168

31. Grundke-lqbal I et al (1986) Microtubule-associated protein tau. A component of Alzheimer paired helical filaments. J Biol Chem 261(13):6084-6089

32. Grundke-lqbal I et al (1986) Abnormal phosphorylation of the microtubuleassociated protein tau (tau) in Alzheimer cytoskeletal pathology. Proc Natl Acad Sci U S A 83(13):4913-4917

33. GTEx Consortium (2013) The Genotype-Tissue Expression (GTEx) project. Nat Genet 45(6):580-585

34. Guo H et al (2004) Active caspase-6 and caspase-6-cleaved tau in neuropil threads, neuritic plaques, and neurofibrillary tangles of Alzheimer's disease. Am J Pathol 165(2):523-531

35. Guo T, Noble W, Hanger DP (2017) Roles of tau protein in health and disease. Acta Neuropathol 133(5):665-704

36. Gyure KA et al (2001) Intraneuronal abeta-amyloid precedes development of amyloid plaques in Down syndrome. Arch Pathol Lab Med 125(4):489-492

37. Hanger DP et al (1991) Tau in Alzheimer's disease and Down's syndrome is insoluble and abnormally phosphorylated. Biochem J 275(Pt 1):99-104

38. Hartley D et al (2015) Down syndrome and Alzheimer's disease: common pathways, common goals. Alzheimers Dement 11(6):700-709

39. Higginbotham L et al (2019) Integrated Proteomics Reveals Brain-Based Cerebrospinal Fluid Biomarkers in Asymptomatic and Symptomatic Alzheimer's Disease, p 806752

40. Hsieh YC et al (2019) Tau-Mediated Disruption of the Spliceosome Triggers Cryptic RNA Splicing and Neurodegeneration in Alzheimer's Disease. Cell Rep 29(2):301-316.e10

41. li K et al (1993) Abnormal distribution of cathepsin proteinases and endogenous inhibitors (cystatins) in the hippocampus of patients with Alzheimer's disease parkinsonism-dementia complex on Guam, and senile dementia and in the aged. Virchows Arch A Pathol Anat Histopathol 423(3):185-194

42. Iqbal $\mathrm{K}$ et al (2010) Tau in Alzheimer disease and related tauopathies. Curr Alzheimer Res 7(8):656-664

43. Irwin DJ (2016) Tauopathies as clinicopathological entities. Parkinsonism Relat Disord 22(Suppl 1):S29-S33

44. Johnson ECB et al (2018) Deep proteomic network analysis of Alzheimer's disease brain reveals alterations in RNA binding proteins and RNA splicing associated with disease. Mol Neurodegener 13(1):52

45. Kamenetz $\mathrm{F}$ et al (2003) APP processing and synaptic function. Neuron 37(6):925-937
46. Kim N et al (2016) Integrated genomic approaches identify upregulation of SCRN1 as a novel mechanism associated with acquired resistance to erlotinib in PC9 cells harboring oncogenic EGFR mutation. Oncotarget 7(12): 13797-13809

47. Kovacs GG, Lee VM, Trojanowski JQ (2017) Protein astrogliopathies in human neurodegenerative diseases and aging. Brain Pathol 27(5):675-690

48. Kovacs GG et al (2016) Aging-related tau astrogliopathy (ARTAG): harmonized evaluation strategy. Acta Neuropathol 131(1):87-102

49. Lane CA, Hardy J, Schott JM (2018) Alzheimer's disease. Eur J Neurol 25(1): 59-70

50. Lin S et al (2015) Secernin-1 contributes to colon cancer progression through enhancing matrix metalloproteinase-2/9 exocytosis. Dis Markers 2015:230703

51. Lindhout FW et al (2019) VAP-SCRN1 interaction regulates dynamic endoplasmic reticulum remodeling and presynaptic function. EMBO J 38(20):e101345

52. Lott IT, Head E (2019) Dementia in Down syndrome: unique insights for Alzheimer disease research. Nat Rev Neurol 15(3):135-147

53. Magistri $\mathrm{M}$ et al (2015) Transcriptomics profiling of Alzheimer's disease reveal neurovascular defects, altered amyloid-beta homeostasis, and deregulated expression of long noncoding RNAs. J Alzheimers Dis 48(3): 647-665

54. Mathys $\mathrm{H}$ et al (2019) Single-cell transcriptomic analysis of Alzheimer's disease. Nature 570(7761):332-337

55. Miyoshi $\mathrm{N}$ et al (2010) SCRN1 is a novel marker for prognosis in colorectal cancer. J Surg Oncol 101(2):156-159

56. Montine TJ et al (2012) National Institute on Aging-Alzheimer's Association guidelines for the neuropathologic assessment of Alzheimer's disease: a practical approach. Acta Neuropathol 123(1):1-11

57. Mori C et al (2002) Intraneuronal Abeta42 accumulation in Down syndrome brain. Amyloid 9(2):88-102

58. Oddo S et al (2003) Triple-transgenic model of Alzheimer's disease with plaques and tangles: intracellular Abeta and synaptic dysfunction. Neuron 39(3):409-421

59. Pennington K et al (2008) Prominent synaptic and metabolic abnormalities revealed by proteomic analysis of the dorsolateral prefrontal cortex in schizophrenia and bipolar disorder. Mol Psychiatry 13(12):1102-1117

60. Ping $L$ et al (2018) Global quantitative analysis of the human brain proteome in Alzheimer's and Parkinson's disease. Sci Data 5:180036

61. Quinn JP et al (2018) Tau proteolysis in the pathogenesis of Tauopathies: neurotoxic fragments and novel biomarkers. J Alzheimers Dis 63(1):13-33

62. Rosler TW et al (2019) Four-repeat tauopathies. Prog Neurobiol 180:101644

63. Sakamoto T et al (2013) A C69-family cysteine dipeptidase from Lactobacillus farciminis JCM1097 possesses strong Gly-pro hydrolytic activity. J Biochem 154(5):419-427

64. Seidler PM et al (2018) Structure-based inhibitors of tau aggregation. Nat Chem 10(2):170-176

65. Seyfried NT et al (2017) A Multi-network Approach Identifies Protein-Specific Co-expression in Asymptomatic and Symptomatic Alzheimer's Disease. Cell Syst 4(1):60-72.e4

66. Siddiqua A, Margittai M (2010) Three- and four-repeat tau coassemble into heterogeneous filaments: an implication for Alzheimer disease. J Biol Chem 285(48):37920-37926

67. Suda $T$ et al (2006) Identification of secernin 1 as a novel immunotherapy target for gastric cancer using the expression profiles of cDNA microarray. Cancer Sci 97(5):411-419

68. Tabaton $\mathrm{M}$ et al (2010) Signaling effect of amyloid-beta(42) on the processing of AbetaPP. Exp Neurol 221(1):18-25

69. Townsend M, Mehta T, Selkoe DJ (2007) Soluble Abeta inhibits specific signal transduction cascades common to the insulin receptor pathway. J Biol Chem 282(46):33305-33312

70. Uhlen $\mathrm{M}$ et al (2015) Proteomics. Tissue-based map of the human proteome. Science 347(6220):1260419

71. Wang $Y$ et al (2009) Tau fragmentation, aggregation and clearance: the dual role of lysosomal processing. Hum Mol Genet 18(21):4153-4170

72. Wang YP et al (2007) Stepwise proteolysis liberates tau fragments that nucleate the Alzheimer-like aggregation of full-length tau in a neuronal cell model. Proc Natl Acad Sci U S A 104(24):10252-10257

73. Way $\mathrm{G}$ et al (2002) Purification and identification of secernin, a novel cytosolic protein that regulates exocytosis in mast cells. Mol Biol Cell 13(9): 3344-3354 
74. Weingarten MD et al (1975) A protein factor essential for microtubule assembly. Proc Natl Acad Sci U S A 72(5):1858-1862

75. Yan SD et al (2009) RAGE and Alzheimer's disease: a progression factor for amyloid-beta-induced cellular perturbation? J Alzheimers Dis 16(4):833-843

76. Zhang W et al (2019) Novel tau filament fold in corticobasal degeneration, a four-repeat tauopathy, p 811703

77. Zhang Z et al (2014) Cleavage of tau by asparagine endopeptidase mediates the neurofibrillary pathology in Alzheimer's disease. Nat Med 20(11):1254-1262

78. Zhao $X$ et al (2016) Caspase-2 cleavage of tau reversibly impairs memory. Nat Med 22(11):1268-1276

\section{Publisher's Note}

Springer Nature remains neutral with regard to jurisdictional claims in published maps and institutional affiliations.

Ready to submit your research? Choose BMC and benefit from:

- fast, convenient online submission

- thorough peer review by experienced researchers in your field

- rapid publication on acceptance

- support for research data, including large and complex data types

- gold Open Access which fosters wider collaboration and increased citations

- maximum visibility for your research: over $100 \mathrm{M}$ website views per year

At BMC, research is always in progress.

Learn more biomedcentral.com/submissions 